\title{
The surface element deposition and corrosion behavior study of multi-cutting in the machining of magnesium alloys
}

\author{
Jinkai $\mathrm{Xu}^{1, \mathrm{a}}$, Kui $\mathrm{Xia}^{1, \mathrm{~b}}$ Linshuai Zhang ${ }^{1, \mathrm{c}}$ Zhanjiang $\mathrm{Yu}^{1, \mathrm{~d}}$ and Huadong $\mathrm{Yu}^{1, \mathrm{e}}$ \\ ${ }^{1}$ Changchun University of Science and Technology, Changchun 130022, China; \\ axujinkai2000@163.com, ${ }^{b} x i a k u i c u s t @ 163 . c o m,{ }^{c} 434203824 @ q q . c o m,{ }^{d} 261230500 @ q q . c o m$, \\ Corresponding author: ${ }^{\mathrm{e}}$ yuhuadong@cust.edu.cn
}

Keywords: WEDM-HS, multi-cut, element deposition, carbonization layer, corrosion behavior.

\begin{abstract}
The surface element deposition and corrosion behavior of AZ91D magnesium alloys multi-cut with high speed wire electrical discharge machine (WEDM-HS) is studied in this paper. The microstructure and surface morphology of the WEDM-HS surfaces were examined with scanning electron microscope (SEM). Chemical composition of the fabricated surface was analyzed by energy-dispersive X-ray spectroscope (EDS). Using Tafel polarization curves to evaluate the corrosion resistance. Experimental results show that WEDM-HS multi-cutting process can effectively improve the surface integrity to obtain a flat surface. Carbon and oxygen of the foreign elements were observed after WEDM-HS cutting. Multi-cutting process increased the oxygen element content in the machined surface. The Multi-cutting surface exhibited excellent corrosion resistance property in the $3.5 \mathrm{wt}$. \% $\mathrm{NaCl}$ solution.
\end{abstract}

\section{Introduction}

Magnesium (Mg) alloys have been the focus of rapidly growing interest as lightweight automotive materials in view of their excellent castability, high specific strength and low density. And Mg alloys are used in transport applications, such as in aircraft and automobiles construction [1-3]. Although mg alloys has the previosly mentioned advantanges, its weakness is that its surface property cannot withstand corrosion under circumstances of chloride containing solutions $[4,5]$. For this reason, the unsatisfactory corrosion performance of $\mathrm{Mg}$ alloys has been a major obstacle to their further use in structural applications.

Some research results of surface modifcation by EDM had been reported by Yan [6], researchers examined carbonization phenomenon on the EDM surface. They found that a carbonization layer was formed when using kerosene as the dielectric fluid. In another experiment, researchers used EDM with a green compact electrode of $\mathrm{Al}-\mathrm{Ti}$ alloy to produce a modified layer that contained rich $\mathrm{TiC}$ on the machined surface.

Electric discharge machining (EDM) removes materials by vaporizing and melting caused by the high heat within the discharge column. Similar to EDM theory, WEDM has the same processing properties. High-speed wire electrical discharge machine (WEDM-HS) has become an important part of advanced manufacturing technology due to the advantage of high efficiency. Huang [7] found the surfaces of the steel specimens were alloyed with wire-electrode material in various degrees according to different cutting passes with WEDM. Alloying between wire-electrode and workpiece materials take place in the recast layer, and the passive anodic current density in the anodic polarization curves was decreased. But there are fewer reports on electric spark machining magnesium alloys. The purpose of this article is to integrate the element deposition and corrosion behavior on the magnesium alloys surface by WEDM-HS multi-cutting.

\section{Experiment}

Materials and working condition. The experimental material was widely used one of the industrial rolling of AZ91D magnesium alloys, and the chemical compositions of the alloys are given in Table 1. The machine used in the experiment was the WEDM-HS of Jinma DK7732-type, the 
working fluid was JR3A emulsion solution, a molybdenum wire-electrode with a diameter of $0.12 \mathrm{~mm}$, and the positive polarity machining mode was employed in this study. The operating parameters for rough-cutting process: The pulse duration was $11 \mu \mathrm{s}$, the pulse interval was $88 \mu \mathrm{s}$, power tube numbers at 2, Wire speed at $60 \mathrm{~Hz}$, The operating parameters for multi-cutting process: The pulse duration was $1 \mu \mathrm{s}$, the pulse interval was $4 \mu \mathrm{s}$, power tube number at 1 , Wire speed at $50 \mathrm{~Hz}$. Feed depth of cut was increased $50 \mu \mathrm{m}$ in multi-cutting process. AZ91D magnesium alloys were sliced into $3 \mathrm{~mm} \times 10 \mathrm{~mm} \times 15 \mathrm{~mm}$ as samples, the operating parameters for cutting process are shown in Table 1, and numbered of samples. After processing the samples soaked in kerosene for 5 minutes and removed debris with brush. And then all samples ultrasonically degreased in acetone for 5 min, followed by ultrasonication with ethanol for $5 \mathrm{~min}$, and dried in hot air immediately.

Table 1 Composition of AZ91D Mg alloy

\begin{tabular}{cccccccc}
\hline Composition & $\mathrm{Al}$ & $\mathrm{Zn}$ & $\mathrm{Mn}$ & $\mathrm{Si}$ & $\mathrm{Fe}$ & $\mathrm{Ni}$ & $\mathrm{Mg}$ \\
\hline Weight (\%) & 8.534 & 0.522 & 0.208 & 0.016 & 0.002 & $<0.001$ & Remainder
\end{tabular}

Surfaces test. Utilizing scanning electron microscope (JSM-6700F) described the microstructure of samples. Chemical composition of the cut surface was determined by energy-dispersive X-ray spectrometer (EDS). Hydrophobic property was tested by water contact angles. Electrochemical tests were performed by CS310 electrochemical workstation. A three-electrode configuration was employed in this electrochemical tests, the sample area was exposed $0.5 \mathrm{~cm}^{2}$ as the working electrode, a platinum electrode as the counter electrode and a saturated calomel electrode as reference electrode. The samples were immersed in the $3.5 \mathrm{wt} \% \mathrm{NaCl} 18 \pm 1{ }^{\circ} \mathrm{C}$ solution without stirring for $5 \mathrm{~min}$ allowed the open circuit potential (OCP) of samples reached to stable values. All experimental chemical reagents were analytically pure reagents, solution was deionized water. For polarization measurements, the potential was scanned with a scan rate of $0.5 \mathrm{mV} / \mathrm{s}$.

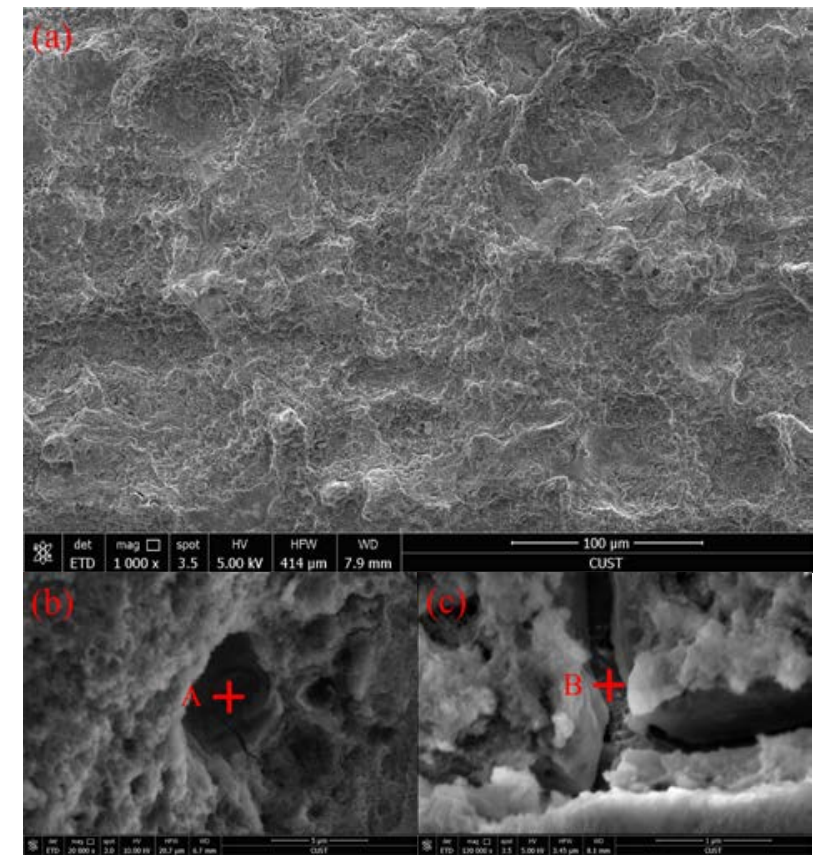

Fig. 1 SEM micrograph of the multi-cutting sample

\section{Results and discussions}

Surface morphology and chemical composition. Fig. 1 displays the SEM microphotograph of WEDM-HS machined surface after different cutting passes. As shown in Fig. 1a, a rough and porous morphology can be observed on the rough machining surface. According to the rough cutting parameter, AZ91D material was removed with higher pulse duration and power tube numbers. The large erosion craters were left on the machined surface, and we can kwon that it has big surface roughness values. Because of the high discharge energy, the surface was seriously damage. Fig. $1 \mathrm{~b}$ 
and Fig. 1c display the local magnification of rough cutting surface (Fig. 1a). The typical defect of WEDM-HS surface micro-cracks and micro-pits are present in Fig. 1b and Fig. 1c respectively.

(a)

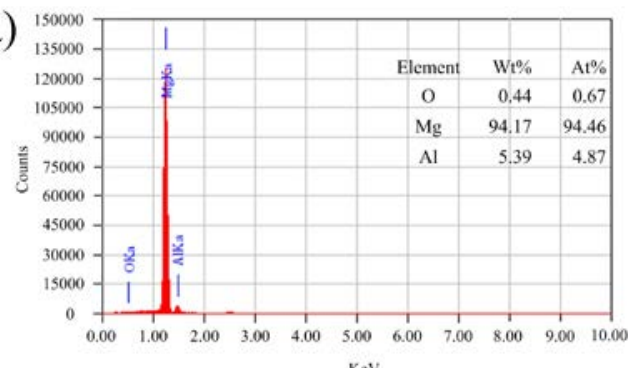

(c)

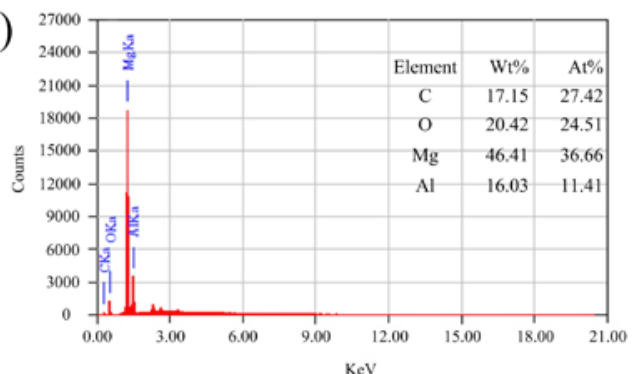

(b)

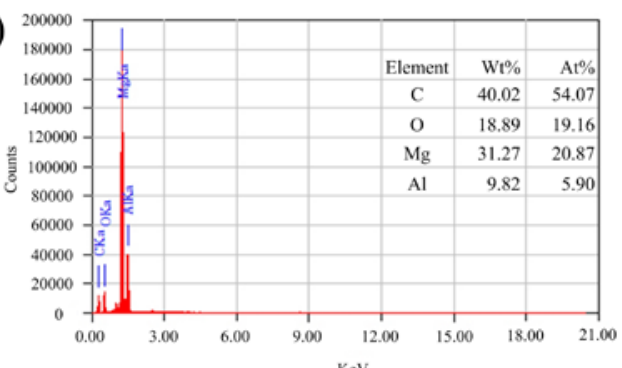

(d)

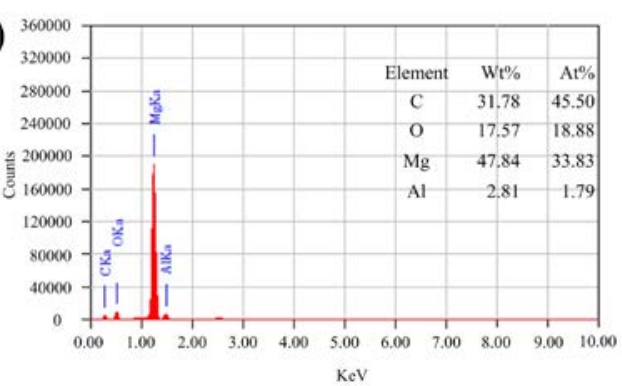

Fig. 2 (a) The EDS analysis of AZ91D matrix and (b) the rough-cutting sample; (c), (d) EDS analysis at the position A, B indicated in Fig. 1a

Fig. 2 shows the EDS analysis results of the AZ91D matrix and WEDM-HS cut surfaces. A typical AZ91D matrix EDS analysis is shown in Fig. 2a, the main chemical elements of matrix were Mg and $\mathrm{Al}$ element. However, Carbon and oxygen of the foreign elements were observed after WEDM-HS cutting (Fig. 2b). The elements of Mg and aluminum were significantly reduced in contrast to matrix. Therefore, we believe that it is possible to produce a carbon layer during processing. Generally, in the case of the emulsion as coolant, the cooling liquid is affected by high temperature in the discharge region, and became gas immediately. During the processing, the discharge of black carbon and other material coagulation attached on the processing surface, lead to the processing surface present dark color. Finally, due to the effect of cooling and solidification, causing carbonization layer formed on the material surface [8]. Fig. 2c and Fig. 2d respectively were the EDS analysis results on specific position $\mathrm{A}$ and $\mathrm{B}$. Although the rough-cutting surface was seriously damage. In which elements carbon and oxygen were also detected in the bottom of micro-cracks and micro-pits. It indicates the carbonization to occur in infinitesimal area, or even nano level and smaller regions.
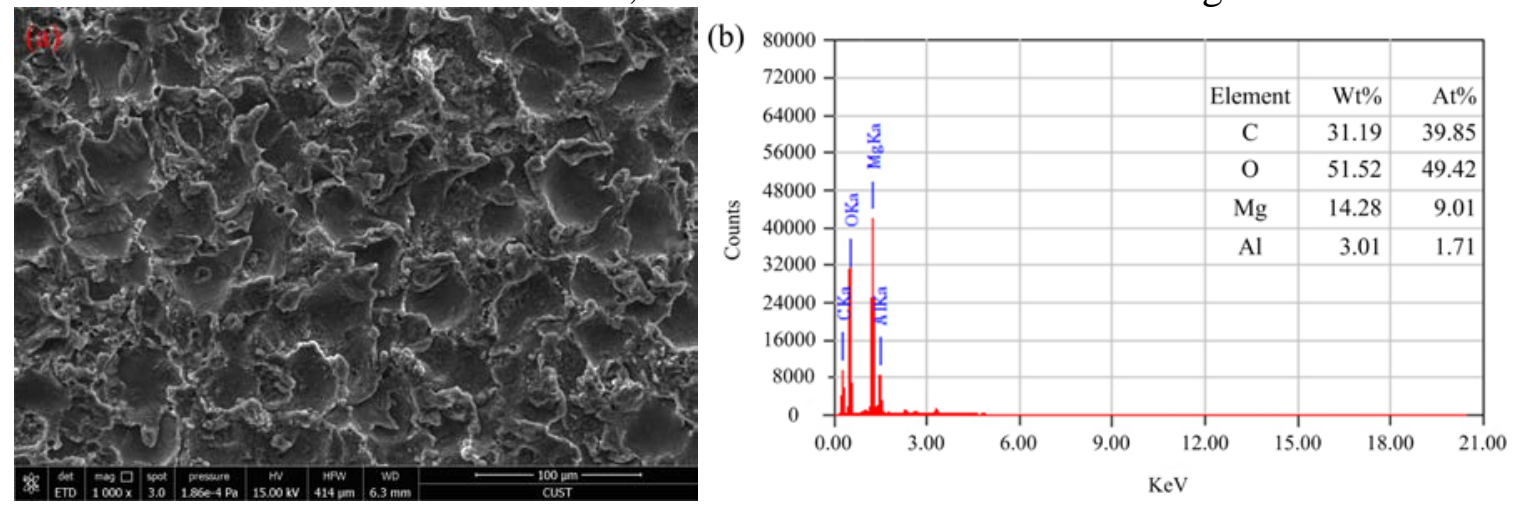

Fig. 3(a) SEM micrograph of the multi-cutting sample, (b) the EDS analysis of multi-cutting sample

Fig. 3a displays the SEM microphotograph of WEDM-HS machined surface after multi-cutting process. The craters size on the machined surface was obviously decreased compared with rough-cutting. Furthermore, the surface integrity was improved signally. At the same magnification, we found that there were fewer micro-cracks and micro-pits on multi-cutting surface. This phenomenon demonstrates that using multi-cutting process has a good consequence in surface integrity and quality, even though magnesium alloys has low density, low hardness, and other 
properties. It has good processing quality at the bottom of crater. Use narrow pulse interval to make crater overlap more, and the bottom area of crater was increased relatively in WEDM-HS machined surface.

Fig. 3b presents the chemical elements analysis results of the multi-cutting surface. Comparison of chemical composition elements we found $\mathrm{Mg}$ element was decreased, and the oxygen element was increased slightly. During the multi-cutting process, AZ91D Mg alloys were exposed to air in a maximum extent, and dielectric fluid was relatively sufficient. Due to more fully oxidation as a result of high temperature involved in the process. It leads to a change of the elements of the machined surface. In addition, narrowing the pulse interval plays an important role in the carbonization process. Continuous discharge is contributed to the combination of the foreign elements and matrix material.

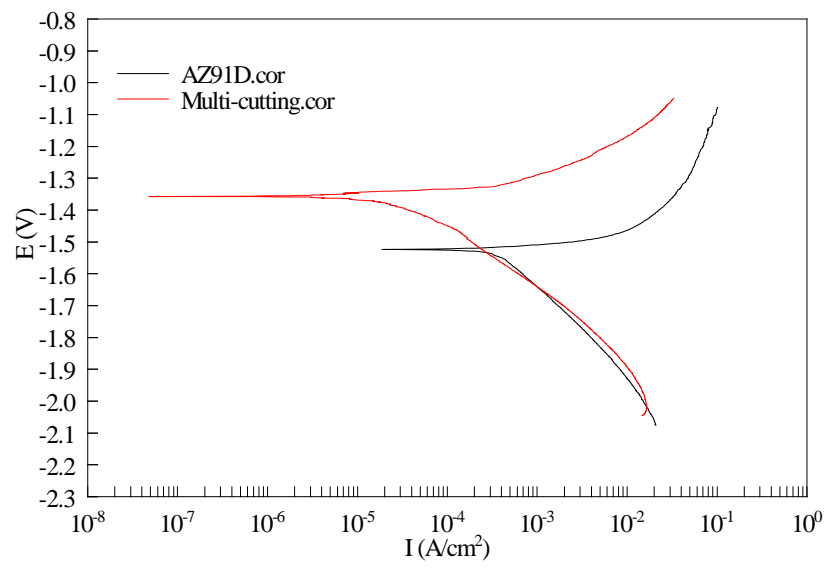

Fig. 4 Potentiodynamic polarization curves of AZ91D matrix and multi-cutting sample

Corrosion measurement. To investigate the corrosion behaviour of AZ91D after carbonization, we used the Tafel polarization curves to evaluate corrosion resistance of the WEDM-HS multi-cutting surface. The Tafel curves of AZ91D with various treatments in the $3.5 \mathrm{wt} . \% \mathrm{NaCl}$ solution are presented in Fig. 4. Using the ground condition as the reference curve, WEDM specimens exhibited a significantly upward shift in curve. The corrosion potential increases from $-1.523 \mathrm{~V}$ of the AZ91D matrix to $-1.356 \mathrm{~V}$ of WEDM-HS machined surface. In the Tafel extrapolation method for measuring the $\mathrm{Mg}$ corrosion rate, the corrosion current density, $\mathrm{i}_{\text {corr }}$ $\left(\mathrm{mA} / \mathrm{cm}^{2}\right)$ is estimated by Tafel extrapolation of the polarization curve, and $i_{\text {corr }}$ is related to the average corrosion rate using $[9,10]$ :

$\mathrm{P}_{\mathrm{i}}=22.85 \times \mathrm{i}_{\text {corr }}$

the corresponding corrosion current densities obtained by Tafel fitting are $3.7 \times 10^{-5} \mathrm{~A} / \mathrm{cm}^{2}$ and $9.3 \times 10^{-4} \mathrm{~A} / \mathrm{cm}^{2}$ for WEDM-HS multi-cutting and AZ91D matrix, So the Pi of the WEDM-HS multi-cutting workpiece $\left(8.45 \times 10^{-4} \mathrm{~mm} / \mathrm{y}\right)$ decreases compared with the AZ91D matrix $\left(2.12 \times 10^{-2} \mathrm{~mm} / \mathrm{y}\right)$. These results indicate that the corrosion resistance was improved obviously after WEDM-HS multi-cutting.

Visible change in current density of the specimens could be attributed to carbonization layer. During the discharge machining process, it can be considered as an Electro-Chemical Machining. The potential of carbon is higher than that of magnesium, and the chemical properties of the oxide is more stable than matrix. As it is clear, the carbonization layer shows the good barrier properties for preventing $\mathrm{Cl}^{-}$and other harmful ions destroyed the surface. This is a reason for an increase in the corrosion resistance of the AZ91D magnesium alloys. Moreover, the results also may be attributed to the machined surface of conventional WEDM in which an amorphous layer caused by rapid cooling was generated to advance the corrosion resistance.

\section{Summary}

Compared with rough-cutting surface, WEDM-HS multi-cutting could obviously improve the surface integrity and decrease micro-cracks and micro-pits on machined surface. The carbon and oxygen of elements were deposited, and this result indicates that the carbonization layer was formed. 
The results of the potentiodynamic polarization curves (Tafel) show that multi-cutting sample with the low corrosion current density. The carbonization layer acts as the barrier prevent $\mathrm{Cl}^{-}$and other harmful ions. That means that the corrosion resistance of the AZ91D was improved.

\section{Acknowledgments}

The authors of the paper acknowledge the support from the National Natural Science Foundation of China (NSFC, No. 51275056 and No. 51305043).

\section{References}

[1]G. Song, A. Atrens, Recent insights into the mechanism of magnesium corrosion and research suggestions, Advanced Engineering Materials. 9 (2007) 177-183.

[2] J. Martin, T. Dominique, L.B. Nathalie, The influence of microstructure on the corrosion ehavior of AZ91D studied by scanning Kelvin probe force microscopy and scanning Kelvin probe, Corrosion Science. 48 (2006) 1193-1208.

[3] S. Deepika, Insights into microstructure based corrosion mechanism of high pressure die cast AM50 alloy, Corrosion Science. 60 (2012) 18-31.

[4] A. Pardo, M.C. Merino, A.E. Coy, R. Arrabal, F. Viejo, E. Matykina, Corrosion $\square$ ehavior of magnesium/aluminium alloys in 3.5 wt.\% NaCl, Corrosion Science. 50 (2008) 823-834.

[5] Y.H. Luo, Y. Sun, J.L. Lv, X.H. Wanga, J. Li, F.S. Wang, Transition of interface oxide layer from porous $\mathrm{Mg}(\mathrm{OH})_{2}$ to dense $\mathrm{MgO}$ induced by polyaniline and corrosion resistance of $\mathrm{Mg}$ alloy therefrom, Applied Surface Science. 328 (2015) 247-254.

[6] Y. C. Lin, B.H. Yan, F.Y. Huang, Surface modification of Al-Zn-Mg aluminum alloy using the combined process of EDM with USM, Journal of Materials Processing Technology. 115 (2001) 359-366.

[7] C.A. Huang, C.L. Shih, K.C. Li, Y.Z. Chang, The surface alloying behavior of martensitic stainless steel cut with wire electrical discharge machine, Applied Surface Science. 252 (2006) 2915-2926.

[8] S.F. Sun, S.Ch. Di, P.X. Lu, D.B. Wei, J. Yu, Y.P. Guo, Microstructure and properties of metamorphic layer formed on Mg-RE alloy in micro-EDM process, Acta Metallurgica Sinica. 49 (2013) 251-156.

[9] G. Song, Recent progress in corrosion and protection of magnesium alloys, Advanced Engineering Materials. 7 (2005) 563-586.

[10] M.C. Zhao, P. Schmutz, S. Brunner, M. Liu, G. Song, A. Atrens, An exploratory study of the corrosion of $\mathrm{Mg}$ alloys during interrupted salt spray testing, Corrosion Science. 51 (2009) 1277-1292. 